\title{
Performance Study of BCH Error Correcting Codes Using the Bit Error Rate Term BER
}

\author{
Elghayyaty Mohamed ${ }^{1}$, Hadjoudja Abdelkader ${ }^{2}$, Omar Mouhib $^{2}$, \\ El Habti El IdrissiAnas ${ }^{2}$, mahjoub chakir ${ }^{1}$ \\ ${ }^{1}$ (Laboratory of the sciences of the engineer and modeling. Faculty of Sciences, University Ibn Tofail Kenitra, \\ Morocco) \\ ${ }^{2}$ (Laboratory of Electrical Engineering and Energy System. Faculty of Sciences, University Ibn Tofail Kenitra, \\ Morocco
}

\begin{abstract}
The quality of a digital transmission is mainly dependent on the amount of errors introduced into the transmission channel. The codes BCH (Bose-Chaudhuri-Hocquenghem) are widely used in communication systems and storage systems. In this paper a Performance study of $\mathrm{BCH}$ error correcting codes is proposed. This paper presents a comparative study of performance between the Bose-Chaudhuri-Hocquenghem codes BCH (15, $7,2)$ and $\operatorname{BCH}(255,231,3)$ using the bit error rate term (BER). The channel and the modulation type are respectively AWGN and PSK where the order of modulation is equal to 2 . First, we generated and simulated the error correcting codes $\mathrm{BCH}(15,7,2)$ and $\mathrm{BCH}(255,231,3)$ using Math lab simulator. Second, we compare the two codes using the bit error rate term (BER), finally we conclude the coding gain for a BER $=10-4$.

Keywords: Bose-Chaudhuri-Hocquenghem codes, Math lab simulator, bit error rate, AWGN channel,
\end{abstract} Performance

\section{INTRODUCTION}

Communication technologies are widely used these days. Due to the growing percentage of people using these technologies, methods are needed to increase the transmission rate without reducing the quality [1].Many digital signaling applications in broadcasting use Forward Error Correction, a technique in which redundant information is added to the signal to allow the receiver to detect and correct errors that may have occurred in transmission. There are different types of error correcting codes based on the type of error expected, expected error rate of the communication medium, and whether re-transmission is possible or not. Few of them are $\mathrm{BCH}$, Turbo, Reed Solomon, Hamming and LDPC. These codes differ from each other in their implementation and complexity[2]. In 1960s Bose,Ray-Chaudhuri, Hocquenghem, independently invented $\mathrm{BCH}$ codes. They are powerful class of cyclic codes with multiple errors correcting capability and well defined mathematical properties. The Galois Field or Finite Field Theory defines the mathematical properties of $\mathrm{BCH}$ codes [3]. The aim of this work is to make a Comparative Performance Analysis of Bose-Chaudhuri-Hocquenghem codes $\operatorname{BCH}(15,7,2)$ and $\operatorname{BCH}(255,231,3)$ using the bit error rate term (BER) and signal energy -to- noise power density ratio $(\mathrm{Eb} / \mathrm{No})$. The rest of the paper is organized as follows. Section II describes the $\mathrm{BCH}$ encoder and illustrates the decoding process. Section III presents a comparison study of performances between $\mathrm{BCH}(15,7,2)$ and $\mathrm{BCH}$ $(255,231,3)$ using the bit error rate (BER). Finally, the concluding remarks are given in Section IV.

\section{BACKGROUND AND RELATED WORK}

\subsection{BCH encoder and $\mathrm{t}<2 \mathrm{~m}-1$ are: \\ $\checkmark$ Block length: $\mathrm{n}=2 \mathrm{~m}-1$ \\ $\checkmark$ Parity check bits: $\mathrm{n}-\mathrm{k}<=\mathrm{mt}$ \\ $\checkmark$ Minimum distance: $\mathrm{d}>=2 \mathrm{t}+1$}

BCH (Bose - Chaudhuri - Hocquenghem) Codes form a large class of multiple random errorcorrecting codes. BCH Code is a generalized form of Hamming Code. The possible BCH codes for $\mathrm{m}>=3$

Achieving the $\mathrm{BCH}$ codes is as follows:

\section{Build the body of the Galois GF (qm)}

Error correcting codes operate over a large extent on powerful algebraic structures called finite fields. A finite field is often known as Galois field after Pierre Galois, the French mathematician. A field is one in which addition, subtraction, multiplication and division can be performed on the field elements and thereby obtaining another element within the set itself. A finite field always contains a finite number of elements and it must be a prime power, say $q=p r$, where $p$ is prime. There exists a field of order $\mathrm{q}$ for each prime power $\mathrm{q}=\mathrm{pr}$ and it is 
unique. In Galois field $\mathrm{GF}(\mathrm{q})$, the elements can take this q different values.

We are exploiting the following properties of a finite field:

a. Addition and multiplication operations are defined.

b. The result of addition or multiplication of two elements is always an element in the field.

c. Zero is an element in the field, such that $\mathrm{a}+0=$ a for any element a in the field.

d. Unity is an element in the field, such that $\mathrm{a} \cdot 1=$ a for any element a in the field.

2. Determine a primitive polynomial using the nth root $\alpha$ in the Galois field GF (qm)

3. Choose $2 t=\gamma-1 \alpha$ consecutive power.

4. Build generator polynomial $\mathrm{g}(\mathrm{x})$ as the least common multiple LCM of minimal polynomials associated with the power to choose $\alpha$.

\subsection{BCH decoder}

Three main steps for $\mathrm{BCH}$ decoding are represented as follows [4]:

- Step1: Computation of syndromes.

- Step2: Berlekamp-Massey algorithm.

- Step3: Detection of error position using Chien Search Block.

\section{a. Syndrome Block}

The BCH code is characterized as $(\mathrm{n}, \mathrm{k}, \mathrm{t})$, where $\mathrm{n}$ is the code length, $\mathrm{k}$ is the data length, and $\mathrm{t}$ is the error correction capability. The n-bit code word $\left(\mathrm{r}_{0}, \mathrm{r}_{1}, \ldots, \mathrm{r}_{\mathrm{n}-1}\right)$ can be interpreted as a received polynomial [5], $\mathrm{R}(\mathrm{x})=$ $r_{0}+r_{1} x^{1}+r_{2} x^{2}+\cdots+r_{n-1} x^{(n-1)} \cdot$ In Syndrome Calculation, 2t syndromes are computed using the following equation:

$\mathrm{S}_{\mathrm{i}}=\mathrm{R}\left(\alpha^{\mathrm{i}}\right)=\sum_{\mathrm{j}=0}^{\mathrm{n}-1} \mathrm{r}_{\mathrm{i}} \alpha^{\mathrm{i} \mathrm{i}}=$

$\mathrm{r}_{0}+\mathrm{r}_{1} \alpha^{\mathrm{i}}+\mathrm{r}_{2} \alpha^{2 \mathrm{i}}+\cdots+\mathrm{r}_{\mathrm{n}-1} \alpha^{\mathrm{i}(\mathrm{n}-1)}$

The syndrome calculator block provides two results. The first result is whether the received code word is correct. The second result provides the syndrome polynomial which will be used to correct the code word if the code word is erroneous. The most common algorithm to perform the syndrome calculation needs $2 \mathrm{t}$ basic cells as defined in Fig.1. Where $1^{\leq} \leq 2 \mathrm{t}$.For each Syndrome $\mathrm{Si}$, where ${ }_{1} \leq_{\mathrm{i}} \leq 2 \mathrm{t}, \mathrm{n}$ iterations or computations are needed to compute the Syndrome Polynomial. All the syndrome coefficients will be equal to 0 if the received code word is correct with at least one coefficient different from 0 if the code word is not correct [6].

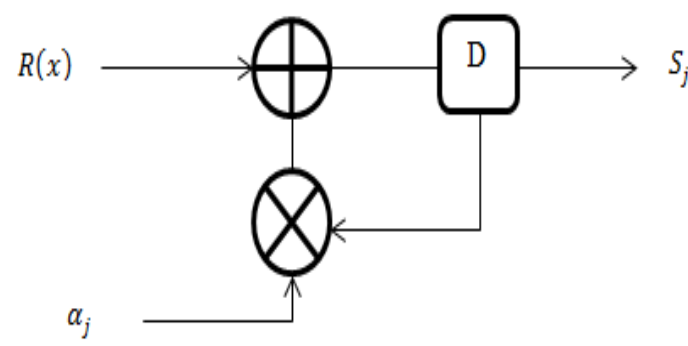

Figure.1. Basic syndrome calculator cell

\section{b. Berlekamp-Massey algorithm}

Berlekamp-Massey algorithm consists of a series of steps based on improving an approximation to the error locator polynomial $\Lambda$ (x) using a correction polynomial $\mathrm{C}(\mathrm{x})$ and the Syndrome values $\mathrm{Si}$ as inputs where: $1 \leq \mathrm{i} \leq 2 \mathrm{t}$. It also requires a step parameter $\mathrm{K}$ and a parameter $\mathrm{L}$ which tracks the order of the equation.

\section{c. Chien Search Block}

This algorithm can detect the error position by calculating $\Lambda\left(\alpha^{-\mathrm{i}}\right)$ where $0 \leq \mathrm{i} \leq \mathrm{n}-1$, such as $\Lambda$ $(\mathrm{x})$ is the error locator polynomial, calculated with the Euclidean algorithm defined in Fig.2. For the case of RS (n, k) we must calculate: $\Lambda\left(\alpha^{-(n-1)}\right), \Lambda\left(\alpha^{-(n-2)}\right) \ldots \Lambda\left(\alpha^{-1}\right), \Lambda\left(\alpha^{-0}\right)$

If the expression reduces to $0, \Lambda\left(\alpha^{-1}\right)=0$, then that value of $\mathrm{x}$ is a root and identifies the error position, else the position does not contain an error [7].

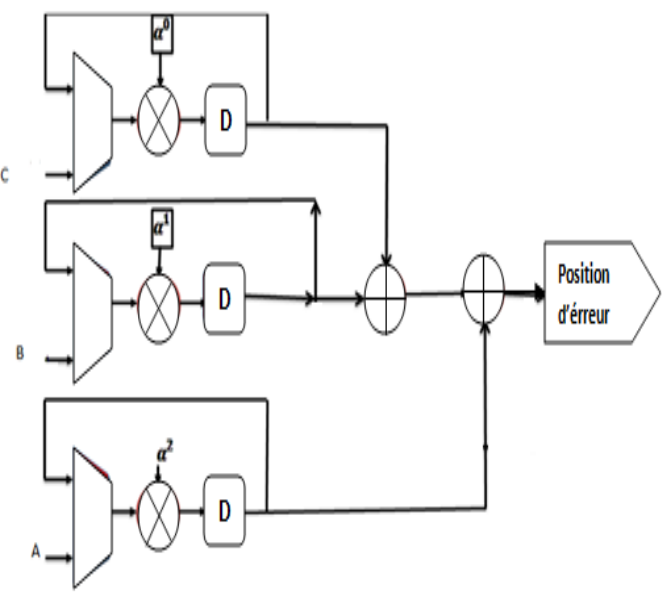

Figure.2. Scheme of Chien Search Block

\section{PERFORMANCE STUDY OF BCH CODES}

To analyze the performance using the bit error rate term (BER) as shown in figure 3, two codes are presented; $\mathrm{BCH}(15,7,2)$ and $\mathrm{BCH}(255$, $231,3)$. The channel and the modulation type are respectively AWGN and PSK where the order of modulation is equal to 2 . 


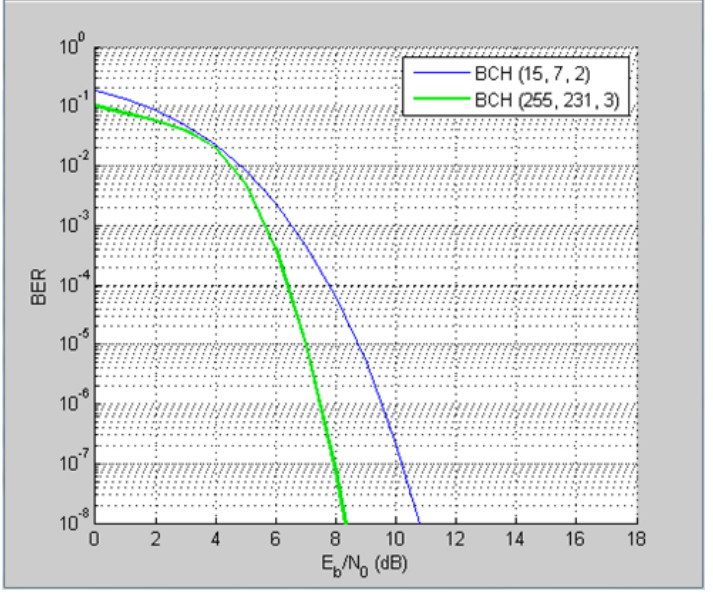

Fig.3. performance of $\mathrm{BCH}(15,7,2)$ and $\mathrm{BCH}$ $(255,231,3)$ codes using the bit error rate term (BER)

The figure 3 shows the performance of two codes: $\mathrm{BCH}(15,7,2)$ and $\mathrm{BCH}(255,231,3)$. One of length 15 , and the other of length 255 , it is observed that the coding gain is $1,4 \mathrm{~dB}$ for a BER = $10^{-4}$ when the length of the code word changes from 15 to 255 . However, when the code word length increases, the complexity of calculating and implementation increases too.

\section{CONCLUSION}

In this paper, we have presented a background and related work of the BoseChaudhuri-Hocquenghem encoder and decoder. This work is based on a simple comparison of codes $\mathrm{BCH}$ $(15,7,2)$ and $\mathrm{BCH}(255,231,3)$. This study addressed the performance of $\mathrm{BCH}$ codes using the bit error rate term (BER) and the noise power density ratio of the signal energy ( $\mathrm{Eb} / \mathrm{No})$.

\section{REFERENCES}

[1]. P. Shrivastava, UP. Singh, Error Detection and Correction Using Reed-Solomon Codes, International Journal of Advanced Research in Computer Science and Software Engineering, 3(8), 2013, 965-969.

[2]. BBC Research and Development, ReedSolomon error correction, British Broadcasting Corporation, 2002.

[3]. Priya Mathew, Lismi Augustine, Sabarinath $\mathrm{G}$ and Tomson Devis, "Hardware implementation of $\mathrm{BCH}(63,51)$ encoder and decoder for WBAN using LFSR and BMA",

[4]. El habti El idrissi Anas, ElgouriRachid, Ahmed Lichioui and HlouLaamari,"Performance study and synthesis of new Error Correcting Codes $\mathrm{RS}, \mathrm{BCH}$ and LDPC Using the Bit Error
Rate (BER) and Field-Programmable Gate Array FPGA", International Journal of Computer Science and Network Security, 2016.

[5]. Y. Lee, H. YooAnd Ic. Park, Small-Area Parallel Syndrome Calculation For Strong Bch Decoding, IeeeXplore, 2014, 16091612.

[6]. El Habti El Idrissi Anas1, El Gouri Rachid1, 2, Ahmed Lichioui3, Hlou Laamari1, "Conception of a new Syndrome Block for $\mathrm{BCH}$ codes with hardware Implementation on FPGA Card", Int. Journal of Engineering Research and Applications, 2014.

[7]. El Habti El Idrissi Anas1, El Gouri Rachid1, 2, Hlou Laamari1, "FPGA Implementation of A New Chien Search Block for Reed-Solomon Codes RS (255, 239) Used In Digital Video Broadcasting DVB-T”, Int. Journal of Engineering Research and Applications, 2014. 